\title{
Does hypoglycaemia affect the improvement in QoL after the transition to insulin in people with type 2 diabetes?
}

\author{
T. H. Wieringa ${ }^{1,2} \cdot$ M. de Wit ${ }^{1,2} \cdot$ J. W. R. Twisk ${ }^{2,3} \cdot$ F. J. Snoek ${ }^{1,2,4}$
}

Received: 21 February 2017 / Accepted: 7 August 2017 / Published online: 12 August 2017

(C) The Author(s) 2017. This article is an open access publication

\begin{abstract}
Purpose Quality of Life (QoL) of insulin-naïve people with type 2 diabetes mellitus (T2DM) improves after transition to insulin. Little is known about the role of hypoglycaemia in this context. Secondary analyses of the Study of the Psychological Impact in Real care of Initiating insulin glargine Treatment (SPIRIT) aimed to investigate the relationship between hypoglycaemia and QoL when transitioning to insulin.

Methods Insulin-naïve Dutch people with T2DM in suboptimal glycaemic control (HbA1c $>53 \mathrm{mmol} / \mathrm{mol} ; 7.0 \%$ ) on maximum dose of oral glucose-lowering medications were included from 363 primary care practices $(n=911)$. Participants started insulin glargine and completed QoL-questionnaires (WHO-5 Well-being Index (WHO-5; emotional
\end{abstract}

T. H. Wieringa

t.wieringa@vumc.nl

M. de Wit

m.dewit@vumc.nl

J. W. R. Twisk

jwr.twisk@vumc.nl

F. J. Snoek

fj.snoek@vumc.nl

1 Department of Medical Psychology, VU University Medical Center (VUMC), De Boelelaan 1117, 1081 HV Amsterdam, The Netherlands

2 Amsterdam Public Health Research Institute, Amsterdam, The Netherlands

3 Department of Clinical Epidemiology and Biostatistics, VU University Medical Center (VUMC), De Boelelaan 1089a, 1081 HV Amsterdam, The Netherlands

4 Department of Medical Psychology, Academic Medical Center (AMC), Meibergdreef 9, 1100 DD Amsterdam, The Netherlands well-being), Hypoglycaemia Fear Survey-worry scale (HFSw; hypoglycaemia fear) and Diabetes Symptom Checklistrevised (DSC-r; diabetes symptom distress) at baseline, 3 and 6 months follow-up. Linear GEE analyses were used to investigate the association between symptomatic, nocturnal, severe hypoglycaemia (number of episodes during 3 months prior to visit) and QoL over time.

Results $52.5 \%$ men participated, mean age 62.2 years $(\mathrm{SD} \pm 10.92$ ), and median $\mathrm{HbA} 1 \mathrm{c} 67 \mathrm{mmol} / \mathrm{mol}$ (range 61-77) (8.3\%). More symptomatic hypoglycaemic episodes were associated with higher HFS-w and DSC-r scores $(P<0.01)$. Experiencing multiple nocturnal or severe episodes was related to higher symptom distress as well, when compared to no episodes. These associations did not change significantly over time.

Conclusions Hypoglycaemia is associated with lower QoL in terms of hypoglycaemia fear and diabetes symptom distress. The transition to insulin does not affect this relationship, suggesting hypoglycaemia in itself has a detrimental effect on diabetes-related QoL independent of treatment regimen.

Keywords Diabetes symptom burden - Emotional wellbeing $\cdot$ Hypoglycaemia $\cdot$ Hypoglycaemia fear $\cdot$ Insulin initiation $\cdot$ Quality of life
Abbreviations
DSC-r Diabetes Symptom Checklist-revised
HbA1c Haemoglobin A1c
HFS-w Hypoglycaemia Fear Survey-worry subscale
QoL Quality of life
SPIRIT Study of the Psychological Impact in Real care of Initiating insulin glargine Treatment
T2DM Type 2 diabetes mellitus
WHO-5 World Health Organization-5 


\section{Background}

Hypoglycaemia is a common, unpredictable and potentially dangerous side effect of insulin therapy for diabetes [1]. Hypoglycaemia can be characterized as: symptomatic hypoglycaemia (an episode of hypoglycaemia which is self-treated), nocturnal hypoglycaemia (a symptomatic episode which takes place at night) and severe hypoglycaemia (an episode of hypoglycaemia in which assistance from a third party is required).

Hypoglycaemia is inversely related to quality of life (QoL) in people with T2DM [2-4]. There is consensus that insulin therapy and insulin secretagogues (e.g. sulfonylureas, meglitinides) due to their mode of action are the main drivers of hypoglycaemia in T2DM [2]. It is estimated that $51 \%$ of people with T2DM recently commenced on insulin therapy (less than 3 years) experience at least one episode of symptomatic hypoglycaemia per year, while 7\% experience at least one severe hypoglycaemia [1]. Research on the impact of hypoglycaemia on the QoL of people with T2DM suggests a greater depression burden [5]. Lifestyle and daily activities can be hampered by hypoglycaemia as a result of the symptoms negatively affecting performance, and/or as a consequence of worrying about hypoglycaemia leading to avoidant, precautionary or compensatory actions aimed to minimize the risk of hypoglycaemic episodes [6]. Fear of hypoglycaemia in people with T2DM is in itself burdensome and may translate into avoidance behaviours resulting in elevated blood glucose levels, and increased risk of long-term complications [7]. We previously demonstrated that QoL improves in people with T2DM 6 months after initiation of insulin therapy, and even after 3 months for emotional well-being [8]. However, it is unknown whether this holds for those experiencing episodes of hypoglycaemia as well.

Using data from the Study of the Psychological Impact in Real care of Initiating insulin glargine Treatment (SPIRIT) [8], a prospective observational study in routine primary care, we analysed the relationship between hypoglycaemia and QoL in people with T2DM from the moment of transition to insulin glargine onwards. Insulin glargine is a long-acting insulin analogue with a more prolonged, consistent duration of action and a lower risk of hypoglycaemia compared to NPH (humane isophane) insulin [9-11]. The SPIRIT database was chosen because patients transferred from oral treatment to insulin treatment which heightened the risk of hypoglycaemia. We hypothesize that those who experience hypoglycaemia will have a less increase in QoL after transition to insulin compared to those who do not experience hypoglycaemia.

\section{Methods}

\section{Participants and procedure}

We used an observational longitudinal dataset for the analyses obtained from the SPIRIT [8]. Data collection took place between January 2006 and July 2008. This study examined the change in emotional well-being, diabetes symptom distress and fear of hypoglycaemia in Dutch people with T2DM who previously used a maximum dose of oral anti-hyperglycaemic medication and were in suboptimal glycaemic control (Haemoglobin $\mathrm{A}_{1 \mathrm{c}}\left(\mathrm{HbA}_{1 \mathrm{c}}\right)>53 \mathrm{mmol} / \mathrm{mol} ; 7.0 \%$ ) [12]. People who used oral anti-hyperglycaemic agents were recruited from 363 Dutch primary care practices, spread across the Netherlands. General practitioners invited eligible people to participate. Inclusion criteria were: in clinical need of initiating long-acting insulin in accordance with the directive of the Dutch College of General Practitioners (which states that insulin therapy should be initiated if, after treatment with a maximum dose of two oral agents, optimal glycaemic control (HbAlc $>53 \mathrm{mmol} / \mathrm{mol} ; 7.0 \%)$ is not achieved), and the ability to complete questionnaires.

\section{Measures}

Measurements were conducted at baseline (moment of transition to insulin therapy, i.e. the day clinician and person with T2DM agreed on starting insulin therapy) and 3 and 6 months after initiation of insulin glargine.

\section{Quality of life}

Emotional well-being was assessed with the World Health Organization (WHO)-5 Well-being Index, a well-validated instrument in people with diabetes assessing emotional wellbeing experienced in the two preceding weeks [13]. The WHO-5 consists of five positively stated items including positive mood, vitality and general interests. Scores were transformed into $0-100$, with higher scores representing better emotional well-being. A score $<50$ is considered indicative of low mood.

Fear of hypoglycaemia was assessed using the worry subscale of the Hypoglycaemia Fear Survey (HFS-w), which assesses hypoglycaemia fear experienced in the 3 months prior to filling out [7]. HFS-w scores were transformed into a $0-100$ scale, with higher scores indicating more worries about hypoglycaemia.

Diabetes symptom distress was measured using the revised version of the Diabetes Symptom Checklist (DSCr) that has good psychometric properties and assesses diabetes symptom distress experienced in the month prior filling out [14]. The DSC-r consists of 34 items grouped into eight symptom subscales: hyperglycaemia, hypoglycaemia, 
cognitive burden, fatigue, cardiovascular burden, neuropathic pain, neuropathic sensitivity and ophthalmic function [14]. Each item asks about the presence of symptoms and, if any, to the burden of this complaint (to answer on a 5-point scale). Scores are transformed into a 0-100 score. A higher score indicates higher diabetes symptom burden.

\section{Demographic and medical outcomes}

Demographic and clinical data were obtained through selfreport: age, sex, weight, height, diabetes duration (years), previous medication use, diabetes-related complications, comorbidity and level of education.

Hypoglycaemia was self-reported as the number of episodes during 3 months prior to visit and divided into symptomatic hypoglycaemia (defined as an episode of hypoglycaemia which is self-treated by the affected individual), nocturnal (defined as a symptomatic episode which takes place at night) and severe hypoglycaemia (defined as an episode of hypoglycaemia in which assistance from a third party is required).

Glycosylated haemoglobin (HbA1c) was obtained from medical charts.

\section{Statistical analyses}

Generalized Estimating Equations (GEE) analyses were conducted to examine the association between hypoglycaemic episodes and QoL-outcomes over time. For every outcome, a crude and an adjusted analysis was performed. Adjustments were made for age, gender, diabetes duration, $\mathrm{HbA1c}$, body mass index (BMI), level of education, and the number of complications. Additionally, the interaction between time and hypoglycaemic episodes was added to both the crude and the adjusted model. Outcome variables with a skewed distribution were log transformed. Hypoglycaemic episodes were treated as categorical variables. The categories were defined according to the median of non-zero values. Categories for symptomatic hypoglycaemia were "no hypoglycaemia", "1-3 hypoglycaemic episodes" and " $\geq 4$ hypoglycaemic episodes". Categories for nocturnal hypoglycaemia were "no hypoglycaemia", "1-2 hypoglycaemic episodes" and " $\geq 3$ hypoglycaemic episodes". Categories for severe hypoglycaemia were: "no hypoglycaemia", "1 hypoglycaemic episode" and " 2 hypoglycaemic episodes".

Multiple imputation was used for missing data [8]. All the analyses were performed on the imputed dataset. IBM SPSS 20 was used for all analyses. Because of multiple testing, a $P$ value threshold of 0.01 was used for statistical significance.

\section{Results}

A total of 1063 people with T2DM consented to participate in the study, of which 43 were found to already use insulin and 109 were not in suboptimal control (HbA1c $\leq 53 \mathrm{mmol} / \mathrm{mol} ; 7.0 \%$ ) [8]. These subgroups were removed from the analyses, resulting in a sample of 911 people. In the original article of SPIRIT [8], analyses were based on the intention-to-treat principle; persons who withdrew from glargine use $(n=99 ; 11 \%)$ were thus included in the analyses. In the same study, logistic regression analyses revealed that dropout was not selective. More information about this sensitivity analysis can be found in the original article [8]. For the WHO-5, missing data were 18.0 and $43.0 \%$. For the HFS-w, missing data were noted for $28.0 \%$ of the participants at the start up to $50.0 \%$ at 6-month follow-up. For the DSC-r, these percentages were $28.0 \%$ and $50.0 \%$, respectively [8]. Characteristics of the study population are shown in Table 1. Changes in HbA1c, QoL-outcomes and hypoglycaemia together with $P$ values are described in the original article of SPIRIT [8]. Since both HFS-w scores and DSC-r scores were distributed as skewed to the right, they were analysed as log transformed in the GEE analyses.

\section{Symptomatic hypoglycaemia}

Those who experienced one or more hypoglycaemic episodes reported more worries about hypoglycaemia compared to those not reporting any hypoglycaemic episode. The symptom burden of patients who experienced four or more episodes was higher compared to patients reporting fewer to no episodes (Table 2). There were no differences in emotional well-being. Changes in hypoglycaemia fear and symptom burden per symptomatic hypoglycaemia category are graphically illustrated in Figs. 1 and 2, respectively.

\section{Nocturnal hypoglycaemia}

DSC-r scores were significantly higher for those experiencing three or more nocturnal hypoglycaemic episodes compared to those not experiencing any nocturnal hypoglycaemia (Table 3; Fig. 3). No significant changes were found for emotional well-being and hypoglycaemia fear.

\section{Severe hypoglycaemia}

Significantly higher DSC-r scores were found for those experiencing two severe hypoglycaemic episodes compared to those not experiencing any episode (Table 4; Fig. 4). No significant changes were found for emotional well-being and hypoglycaemia fear. 
Table 1 Demographics of the study population and changes in clinical outcomes and QoL during the period of study

\begin{tabular}{|c|c|c|c|}
\hline Demographics & Baseline & 3 months & 6 months \\
\hline$N$ & 911 & & \\
\hline \multicolumn{4}{|l|}{ Gender } \\
\hline Men & $479(52.5 \%)$ & & \\
\hline Women & $432(47.5 \%)$ & & \\
\hline Age (years) & $62.15 \pm 10.92$ & & \\
\hline \multicolumn{4}{|l|}{ Level of education } \\
\hline Low & $626(68.7 \%)$ & & \\
\hline Average & $188(20.7 \%)$ & & \\
\hline High & $97(10.7 \%)$ & & \\
\hline Diabetes duration (years) & $6.00(3.00-9.00)$ & & \\
\hline \multicolumn{4}{|l|}{$\mathrm{HbA} 1 \mathrm{c}(\mathrm{mmol} / \mathrm{mol})^{\mathrm{a}}$} \\
\hline Mean \pm SD & $72 \pm 17$ & $61 \pm 11$ & $57 \pm 11$ \\
\hline Median (25th-75th) & $67(61-77)$ & $60(53-67)$ & $56(50-63)$ \\
\hline \multicolumn{4}{|l|}{$\operatorname{HbA} 1 \mathrm{c}(\%)^{\mathrm{a}}$} \\
\hline Mean \pm SD & $8.7 \pm 1.5$ & $7.7 \pm 1.0$ & $7.4 \pm 1.0$ \\
\hline Median (25th-75th) & $8.3(7.7-9.2)$ & $7.6(7.0-8.3)$ & $7.3(6.7-7.9)$ \\
\hline \multicolumn{4}{|c|}{ Symptomatic hypoglycaemia ${ }^{b}$} \\
\hline 0 episodes & $572(62.8 \%)$ & $524(57.6 \%)$ & $514(56.5 \%)$ \\
\hline 1,2 or 3 episodes & $163(17.9 \%)$ & $211(23.2 \%)$ & $232(25.4 \%)$ \\
\hline 4 or more episodes & $176(19.3 \%)$ & $176(19.3 \%)$ & $165(18.1 \%)$ \\
\hline \multicolumn{4}{|l|}{ Nocturnal hypoglycaemia ${ }^{\mathrm{b}}$} \\
\hline 0 episodes & $783(86.0 \%)$ & $781(85.8 \%)$ & $745(81.8 \%)$ \\
\hline 1 or 2 episodes & $62(6.8 \%)$ & $85(9.4 \%)$ & $110(12.1 \%)$ \\
\hline 3 or more episodes & $66(7.2 \%)$ & $45(4.9 \%)$ & $56(6.1 \%)$ \\
\hline \multicolumn{4}{|l|}{ Severe hypoglycaemia ${ }^{b}$} \\
\hline 0 episodes & $882(96.8 \%)$ & $872(95.7 \%)$ & $860(94.3 \%)$ \\
\hline 1 episode & $14(1.5 \%)$ & $28(3.1 \%)$ & $27(3.0 \%)$ \\
\hline 2 episodes & $15(1.6 \%)$ & $11(1.2 \%)$ & $24(2.6 \%)$ \\
\hline \multicolumn{4}{|l|}{ Previous treatment $\mathrm{c}^{\mathrm{c}}$} \\
\hline SU-derivate & 697 & & \\
\hline Other & 743 & & \\
\hline Body mass index (BMI) & $30.09 \pm 5.85$ & $30.17 \pm 5.80$ & $30.51 \pm 5.76$ \\
\hline \multicolumn{4}{|l|}{ Complications } \\
\hline 0 & $648(71.1 \%)$ & & \\
\hline 1 or more & $263(28.9 \%)$ & & \\
\hline \multicolumn{4}{|l|}{ Complications $^{\mathrm{d}}$} \\
\hline Nephropathy & $21(2.3 \%)$ & & \\
\hline Neuropathy & $86(9.4 \%)$ & & \\
\hline Retinopathy & $42(4.6 \%)$ & & \\
\hline Macroalbuminuria & $26(2.9 \%)$ & & \\
\hline Macroangiopathy & $64(7.0 \%)$ & & \\
\hline Microalbuminuria & $110(12.1 \%)$ & & \\
\hline WHO- $5^{\mathrm{e}}$ & $56.71(25.52)$ & $63.33(21.22)$ & $65.27(20.52)$ \\
\hline HFS-w ${ }^{f}$ & $7.69(1.92-21.15)$ & $5.77(0.00-15.38)$ & $3.85(0.00-15.38)$ \\
\hline DSC- $\mathrm{r}^{\mathrm{g}}$ & $11.71(4.71-22.14)$ & $8.07(3.13-17.14)$ & $8.09(2.88-16.04)$ \\
\hline
\end{tabular}

For dichotomous or categorical variables the absolute numbers by subgroups and the percentage compared to the overall study population are displayed. For normally distributed variables the mean and standard deviation are shown. For skewed variables the median and the 25th and 75th percentile are shown

${ }^{a}$ At baseline HbA1c was skewed distributed, but normally distributed at 3 and 6 months

${ }^{b}$ Number of episodes during 3 months prior to visit

${ }^{\mathrm{c}}$ One case may use multiple oral agents 
Table 1 (continued)

${ }^{\mathrm{d}}$ One case may have multiple complications

${ }^{\mathrm{e}}$ Measured as emotional well-being experienced during 2 weeks prior to visit

${ }^{\mathrm{f}}$ Measured as hypoglycaemia fear experienced during 3 months prior to visit

${ }^{\mathrm{g}}$ Measured as diabetes symptom distress experienced during month prior to visit

Table 2 Association between symptomatic hypoglycaemia and WHO-5, HFS-w and DSC-r

\begin{tabular}{|c|c|c|c|c|c|c|}
\hline & \multicolumn{3}{|l|}{ Unadjusted model } & \multicolumn{3}{|l|}{ Adjusted model $^{\mathrm{a}}$} \\
\hline & Beta & $P$ value & $95 \%-\mathrm{CI}$ & Beta & $P$ value & $95 \%-\mathrm{CI}$ \\
\hline \multicolumn{7}{|c|}{ WHO-5 } \\
\hline $0-1^{\mathrm{b}}$ & 0.33 & 0.565 & -0.79 to 1.44 & -0.27 & 0.673 & -1.53 to 0.99 \\
\hline $1-2^{c}$ & -0.75 & 0.260 & -2.05 to 0.55 & -0.52 & 0.499 & -2.02 to 0.99 \\
\hline \multirow[t]{2}{*}{$0-2^{\mathrm{d}}$} & -0.42 & 0.523 & -1.72 to 0.87 & -0.79 & 0.302 & -2.29 to 0.71 \\
\hline & $\begin{array}{l}\text { Ratio of geometric } \\
\text { averages }\end{array}$ & $P$ value & $95 \%$-CI & $\begin{array}{l}\text { Ratio of geometric } \\
\text { averages }\end{array}$ & $P$ value & $95 \% \mathrm{CI}$ \\
\hline \multicolumn{7}{|l|}{ HFS-w } \\
\hline $0-1^{\mathrm{b}}$ & 1.16 & $<0.001$ & 1.08 to 1.24 & 1.24 & $<0.001$ & 1.15 to 1.35 \\
\hline $1-2^{c}$ & 1.04 & 0.321 & 0.96 to 1.13 & 1.07 & 0.174 & 0.97 to 1.17 \\
\hline $0-2^{\mathrm{d}}$ & 1.21 & $<0.001$ & 1.11 to 1.31 & 1.33 & $<0.001$ & 1.20 to 1.47 \\
\hline \multicolumn{7}{|l|}{ DSC-r } \\
\hline $0-1^{\mathrm{b}}$ & 1.01 & 0.721 & 0.96 to 1.06 & 1.04 & 0.240 & 0.98 to 1.10 \\
\hline $1-2^{\mathrm{c}}$ & 1.08 & 0.004 & 1.03 to 1.14 & 1.10 & 0.004 & 1.03 to 1.17 \\
\hline $0-2^{\mathrm{d}}$ & 1.09 & 0.002 & 1.03 to 1.16 & 1.14 & $<0.001$ & 1.06 to 1.22 \\
\hline
\end{tabular}

Hypoglycaemia was self-reported as number of episodes during 3 months prior to visit. WHO-5 was self-reported as emotional well-being experienced during 2 weeks prior to visit; HFS-w as hypoglycaemia fear during 3 months prior to visit; DSC-r as diabetes symptom distress during month prior to visit

${ }^{a}$ Adjusted for age, diabetes duration, HbA1c, body mass index, level of education, the number of complications and gender

${ }^{\mathrm{b}}$ Comparison between group 0 (no hypoglycaemia) and group 1 (1 hypoglycaemic episode) regarding severe hypoglycaemia

${ }^{\mathrm{c}}$ Comparison between group 1 (1 hypoglycaemic episode) and group 2 (2 hypoglycaemic episodes) regarding severe hypoglycaemia

${ }^{\mathrm{d}}$ Comparison between group 0 (no hypoglycaemia) and group 2 (2 hypoglycaemic episodes) regarding severe hypoglycaemia

${ }^{\mathrm{e}} \mathrm{HFS}-\mathrm{w}$ and DSC-r scores were analysed as log transformed and back transformed with a ratio of geometric averages as a result. This can be interpreted as follows: "the geometric average of the reference group ... times greater compared to the geometric average of the compared group"

Fig. 1 Changes in median HFS-w score per symptomatic hypoglycaemia category

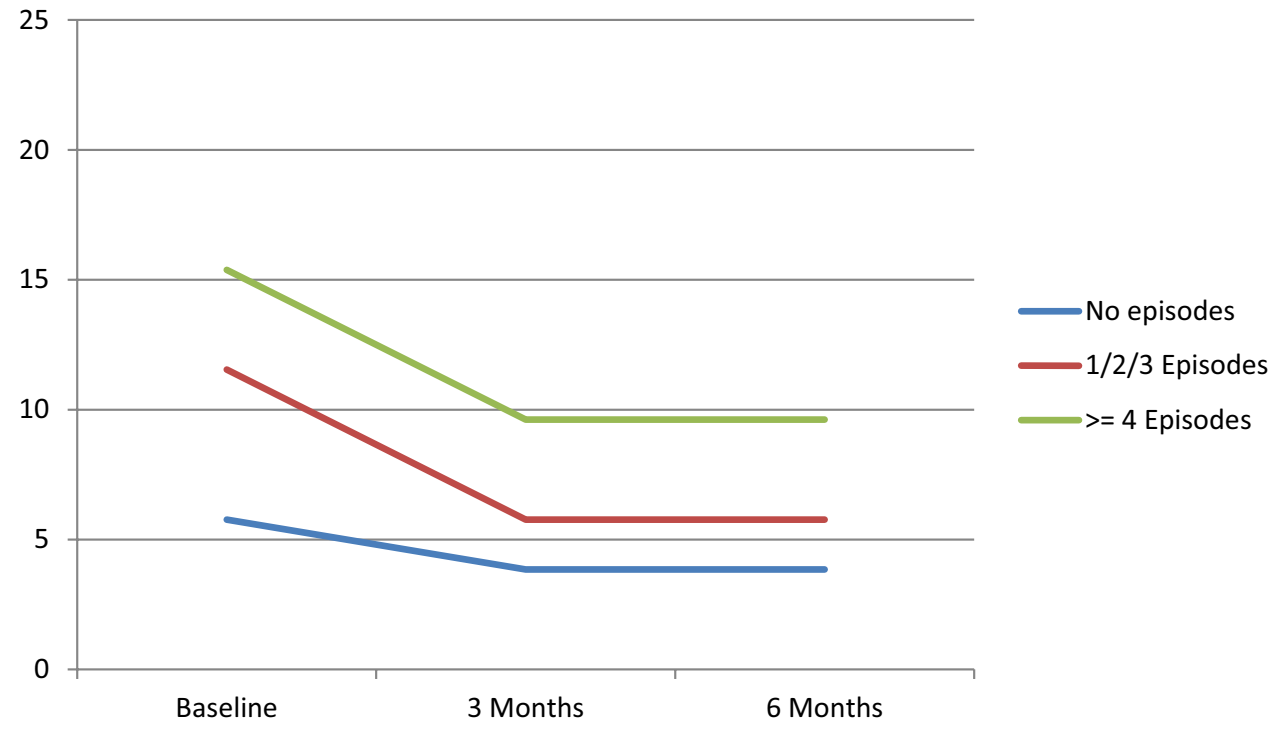


Fig. 2 Changes in median DSC-r score per symptomatic hypoglycaemia category

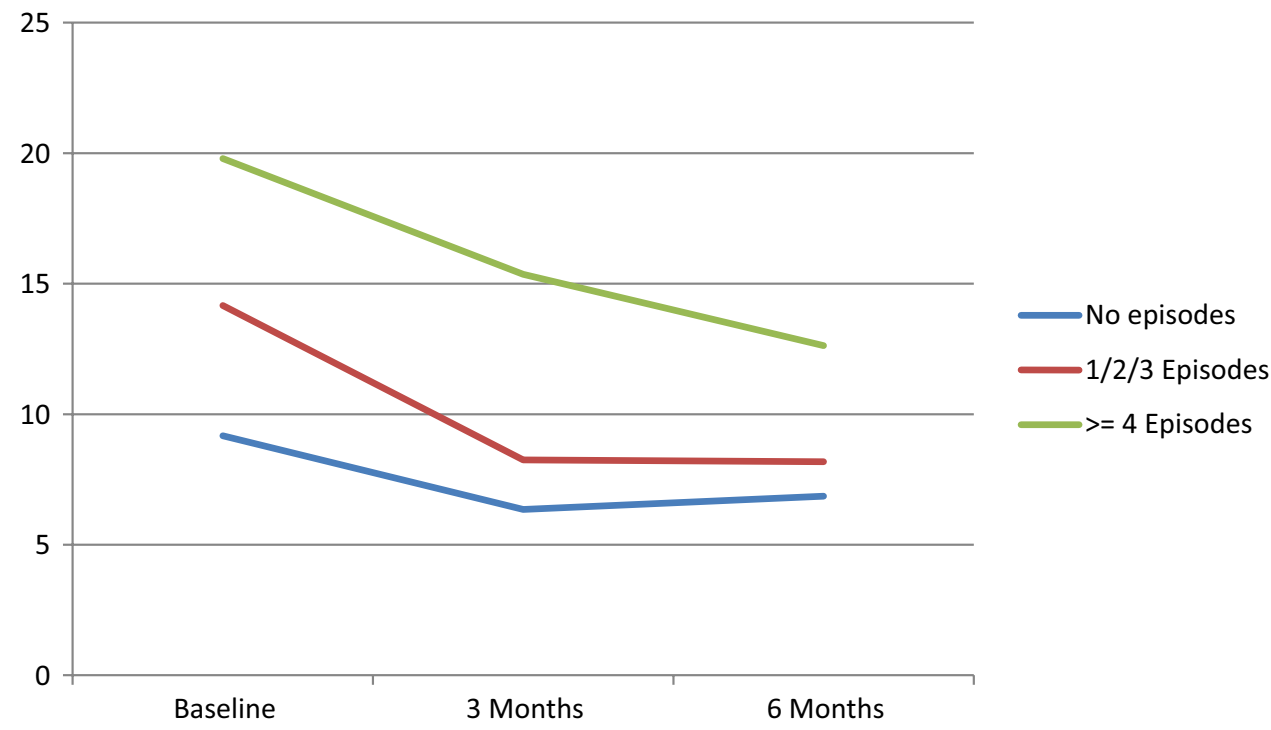

Table 3 Association between nocturnal hypoglycaemia and WHO-5, HFS-w and DSC-r

\begin{tabular}{|c|c|c|c|c|c|c|}
\hline & \multicolumn{3}{|l|}{ Unadjusted model } & \multicolumn{3}{|l|}{ Adjusted model $^{\mathrm{a}}$} \\
\hline & Beta & $P$ value & 95\%-CI & Beta & $P$ value & $95 \%-\mathrm{CI}$ \\
\hline \multicolumn{7}{|c|}{ WHO-5 } \\
\hline $0-1^{\mathrm{b}}$ & 1.15 & 0.093 & -0.19 to 2.50 & 0.62 & 0.423 & -0.90 to 2.14 \\
\hline $1-2^{\mathrm{c}}$ & -3.03 & 0.002 & -1.10 to -4.96 & -1.97 & 0.059 & -4.02 to 0.08 \\
\hline \multirow[t]{2}{*}{$0-2^{\mathrm{d}}$} & -1.88 & 0.027 & -3.53 to -0.22 & -1.35 & 0.154 & -3.20 to 0.51 \\
\hline & $\begin{array}{l}\text { Ratio of geometric } \\
\text { averages }\end{array}$ & $P$ value & $95 \%$-CI & $\begin{array}{l}\text { Ratio of geometric } \\
\text { averages }\end{array}$ & $P$ value & $95 \%-\mathrm{CI}$ \\
\hline \multicolumn{7}{|l|}{ HFS-w } \\
\hline $0-1^{\mathrm{b}}$ & 1.03 & 0.481 & 0.95 to 1.12 & 1.09 & 0.101 & 0.98 to 1 \\
\hline $1-2^{c}$ & 1.09 & 0.145 & 0.97 to 1.22 & 1.04 & 0.574 & 0.91 to 1.19 \\
\hline $0-2^{\mathrm{d}}$ & 1.12 & 0.015 & 1.02 to 1.23 & 1.13 & 0.029 & 1.01 to 1.26 \\
\hline \multicolumn{7}{|l|}{ DSC-r } \\
\hline $0-1^{\mathrm{b}}$ & 1.00 & 0.906 & 0.93 to 1.07 & 1.05 & 0.255 & 0.97 to 1.14 \\
\hline $1-2^{\mathrm{c}}$ & 1.10 & 0.059 & 1.00 to 1.21 & 1.10 & 0.093 & 0.99 to 1.22 \\
\hline $0-2^{\mathrm{d}}$ & 1.09 & 0.029 & 1.01 to 1.18 & 1.15 & 0.004 & 1.05 to 1.26 \\
\hline
\end{tabular}

Hypoglycaemia was self-reported as number of episodes during 3 months prior to visit. WHO-5 was self-reported as emotional well-being experienced during 2 weeks prior to visit; HFS-w as hypoglycaemia fear experienced during 3 months prior to visit; DSC-r as diabetes symptom distress experienced during month prior to visit

${ }^{a}$ Adjusted for age, diabetes duration, HbA1c, body mass index, level of education, the number of complications and gender

${ }^{\mathrm{b}}$ Comparison between group 0 (no hypoglycaemia) and group 1 ( 1 or 2 hypoglycaemic episodes) regarding nocturnal hypoglycaemia

${ }^{\mathrm{c}}$ Comparison between group 1 ( 1 or 2 hypoglycaemic episodes) and group 2 ( 3 or more hypoglycaemic episodes) regarding nocturnal hypoglycaemia

${ }^{\mathrm{d}}$ Comparison between group 0 (no hypoglycaemia) and group 2 (3 or more hypoglycaemic episodes) regarding nocturnal hypoglycaemia

${ }^{\mathrm{e}}$ HFS-w and DSC-r scores were analysed as log transformed, and back transformed with a ratio of geometric averages as a result. This can be interpreted as follows: "the geometric average of the reference group ... times greater compared to the geometric average of the compared group" 
Fig. 3 Changes in median DSC-r score per nocturnal hypoglycaemia category

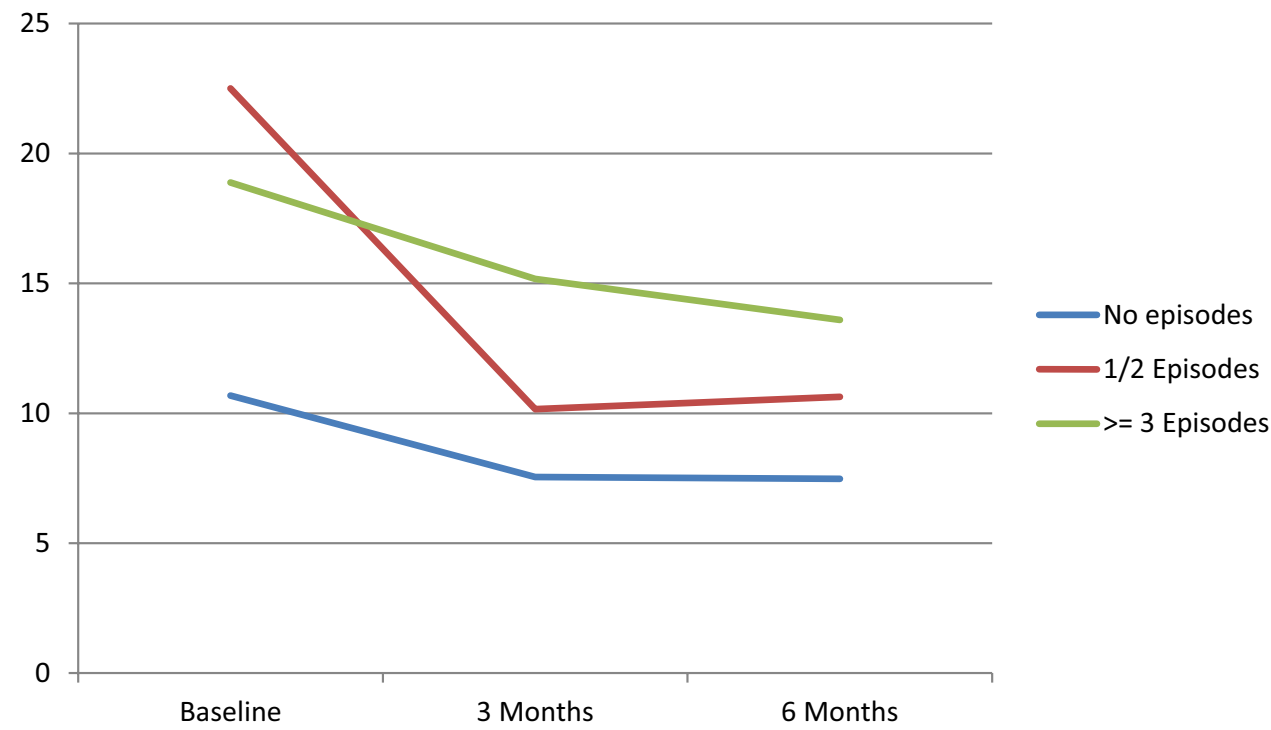

Table 4 Association between severe hypoglycaemia and WHO-5, HFS-w and DSC-r

\begin{tabular}{|c|c|c|c|c|c|c|}
\hline & \multicolumn{3}{|l|}{ Unadjusted model } & \multicolumn{3}{|l|}{ Adjusted model $^{\mathrm{a}}$} \\
\hline & Beta & $P$ value & 95\%-CI & Beta & $P$ value & $95 \%-\mathrm{CI}$ \\
\hline \multicolumn{7}{|c|}{ WHO-5 } \\
\hline $0-1^{\mathrm{b}}$ & 1.45 & 0.214 & -0.84 to 3.73 & 0.96 & 0.517 & -1.94 to 3.86 \\
\hline $1-2^{\mathrm{c}}$ & -1.77 & 0.286 & -5.03 to 1.48 & -2.58 & 0.224 & -6.75 to 1.58 \\
\hline \multirow[t]{2}{*}{$0-2^{\mathrm{d}}$} & -0.33 & 0.801 & -2.87 to 2.21 & -1.63 & 0.305 & -4.73 to 1.48 \\
\hline & $\begin{array}{l}\text { Ratio of geometric } \\
\text { averages }\end{array}$ & $P$ value & $95 \%$-CI & $\begin{array}{l}\text { Ratio of geometric } \\
\text { averages }\end{array}$ & $P$ value & $95 \%-\mathrm{CI}$ \\
\hline \multicolumn{7}{|l|}{ HFS-w } \\
\hline $0-1^{\mathrm{b}}$ & 1.01 & 0.915 & 0.90 to 1.13 & 1.04 & 0.573 & 0.91 to 1.19 \\
\hline $1-2^{c}$ & 1.03 & 0.753 & 0.85 to 1.26 & 1.09 & 0.480 & 0.86 to 1.39 \\
\hline $0-2^{\mathrm{d}}$ & 1.04 & 0.651 & 0.88 to 1.22 & 1.13 & 0.229 & 0.92 to 1.39 \\
\hline \multicolumn{7}{|l|}{ DSC-r } \\
\hline $0-1^{\mathrm{b}}$ & 0.99 & 0.827 & 0.89 to 1.10 & 1.00 & 0.996 & 0.87 to 1.15 \\
\hline $1-2^{\mathrm{c}}$ & 1.12 & 0.101 & 0.98 to 1.27 & 1.16 & 0.068 & 0.99 to 1.37 \\
\hline $0-2^{\mathrm{d}}$ & 1.10 & 0.041 & 1.00 to 1.21 & 1.17 & 0.004 & 1.05 to 1.29 \\
\hline
\end{tabular}

Hypoglycaemia was self-reported as number of episodes during 3 months prior to visit. WHO-5 was self-reported as emotional well-being experienced during 2 weeks prior to visit; HFS-w as hypoglycaemia fear experienced during 3 months prior to visit; DSC-r as diabetes symptom distress experienced during month prior to visit

${ }^{a}$ Adjusted for age, diabetes duration, HbA1c, body mass index, level of education, the number of complications and gender

${ }^{\mathrm{b}}$ Comparison between group 0 (no hypoglycaemia) and group 1 (1,2 or 3 hypoglycaemic episodes) regarding symptomatic hypoglycaemia

${ }^{\mathrm{c}}$ Comparison between group 1 (1,2 or 3 hypoglycaemic episodes) and group 2 (4 or more hypoglycaemic episodes) regarding symptomatic hypoglycaemia

${ }^{\mathrm{d}}$ Comparison between group 0 (no hypoglycaemia) and group 2 (4 or more hypoglycaemic episodes) regarding symptomatic hypoglycaemia ${ }^{e}$ HFS-w and DSC-r scores were analysed as log transformed, and back transformed with a ratio of geometric averages as a result. This can be interpreted as follows: "the geometric average of the reference group times greater compared to the geometric average of the compared group"

In all analyses, the interaction between time and hypoglycaemic episodes was not statistically significant. This implicates that the association between hypoglycaemic events and QoL does not change over time.

\section{Discussion}

This study aimed to assess the association between hypoglycaemia and QoL in people with T2DM on maximum 
Fig. 4 Changes in median DSC-r score per severe hypoglycaemia category

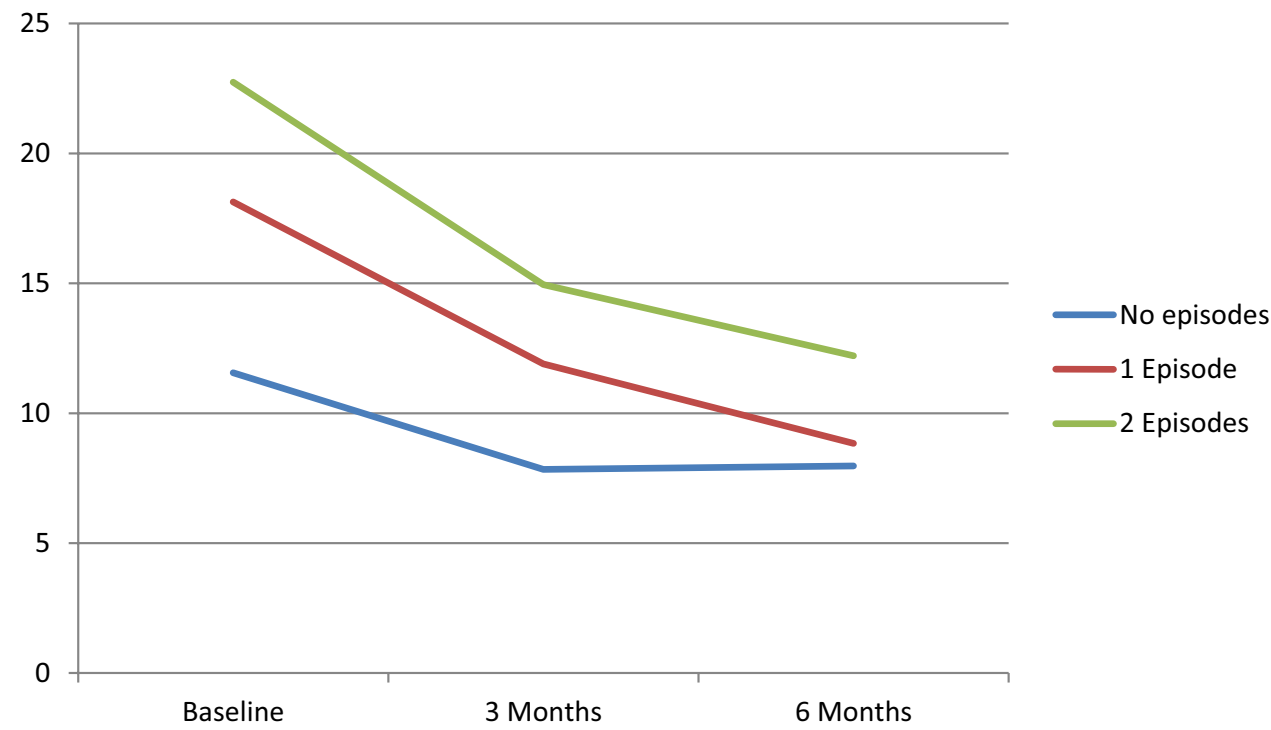

dosage oral blood glucose-lowering medication and examine whether this association changes over time, after transition to insulin glargine. Hypoglycaemia was associated with lower QoL in terms of both hypoglycaemia fear and diabetes symptom distress, the diabetes-related aspects of QoL and not the overall, generic emotional well-being. The initiation of insulin therapy did not affect the relationship between hypoglycaemia and QoL. This suggests that hypoglycaemia in itself has a detrimental effect, independent of treatment regimen.

Previous studies reported severe hypoglycaemia to have a greater impact on QoL compared to symptomatic hypoglycaemia, and nocturnal hypoglycaemia to influence QoL more than daytime episodes [15]. In our study we could not demonstrate these associations, probably due to the low number of people experiencing nocturnal and severe episodes. We did find, in line with previous studies, that frequency of hypoglycaemia is negatively associated with QoL $[3,5,15$, 16]. However, further research is warranted in people with T2DM with higher incidence of severe hypoglycaemia.

The question arises whether the negative impact of hypoglycaemia on diabetes-related QoL found in this study is to be regarded clinically relevant. For diabetes symptom distress we found statistically significant increases in patients experiencing multiple hypoglycaemia episodes (both symptomatic, nocturnal and severe) and for hypoglycaemia fear we found a statistically significant increase in patients experiencing one or more symptomatic episodes compared to those with no symptomatic hypos, but very little is known about the clinical relevance of these measures. This stresses the importance of future research studying the clinical relevance of hypoglycaemia fear and changes in diabetes symptom distress.
Remarkably, hypoglycaemia rates when using oral agents are relatively high and insulin glargine is having a scarce effect on these rates. However, this is in line with previous studies [1]. The UK Hypoglycaemia Study Group (2007) found no difference in T2DM patients treated with sulfonylureas (SUs) or insulin for less than two years in both the proportion experiencing severe hypoglycaemia and the proportion experiencing mild symptomatic hypoglycaemia over a 9-12-month period [1]. A large proportion of patients in our study [76,51\% (697 patients)] used SU-derivates before changing to glargine. Furthermore, Marrett et al. estimated $63 \%$ of the T2DM patients on oral medication experiencing one or more self-reported hypoglycaemic events per half a year [3]. As insulin glargine provides a more prolonged, consistent duration of action and a lower risk of hypoglycaemia compared to NPH (humane isophane) insulin [3, 4, 17], glycaemic control improves while having a scarce effect on hypoglycaemia rates.

This study has several limitations that are worth mentioning. First, hypoglycaemic episodes were self-reported. This may have resulted in underreporting of hypoglycaemia in our study population, as people with diabetes may not always recall or recognize symptoms of hypoglycaemia [18, 19] or may have limited knowledge about hypoglycaemia itself [20]. However, comparable or even lower prevalence rates of severe hypoglycaemia are found in previous research $[1,21]$. Continuous glucose monitoring (CGM) could help establish the reliability of self-report, but is not feasible in a large observational study in primary care. In addition, the relatively large number of missing data is a potential weakness of observational studies, and confirmed in our study [8]. However, multiple imputation can be viewed as the most robust way of dealing with missing data [22]. 
This study has strengths as well. We used well-validated measures of quality of life, supporting internal validity. The study was conducted in a large and heterogeneous sample of people with T2DM in primary care settings across different regions of the country [8], which favours external validity, i.e. generalisability of our findings. We set the $p$ value threshold at 0.01 to increase the probability of the findings, which can be regarded as a strength of this study as well.

\section{Conclusions}

In conclusion, we found that hypoglycaemic episodes have a negative impact on QoL in terms of both hypoglycaemia fear and diabetes symptom distress; therefore, deserving clinical attention. We observed an impact on both hypoglycaemia fear and diabetes symptom distress with more than one episode. The initiation of insulin glargine does, however, not affect this association. Those experiencing multiple hypoglycaemic episodes report lower diabetes-related QoL compared to those not experiencing hypoglycaemia, regardless of treatment regimen. Future studies should be focused on clinically relevant changes in hypoglycaemia fear and diabetes symptom distress to interpret our findings for clinical practice. Prevention and adequate management of hypoglycaemia remains valuable and should be adequately monitored as well as the QoL of people with T2DM, with as much attention for patients using oral agents as for patients initiating insulin therapy.

Acknowledgements The dataset of the Study of the Psychological Impact in Real care of Initiating insulin glargine Treatment (SPIRIT) is used for this study. The authors wish to express their gratitude to T. R. S. Hajos for enabling us to use this dataset.

Author contribution THW performed the data analyses and drafted the manuscript. MdW, and FJS designed the study and drafted the manuscript. JWRT analysed the data and helped to draft the manuscript. All authors read and approved the final manuscript.

\section{Compliance with ethical standards}

Conflict of interest The authors declare that they have no conflict of interest.

Ethical approval In view of its observational and non-invasive nature, this study was deemed not subject to the Dutch Medical Research Involving Human Subjects Act.

Informed consent Informed consent was obtained from all individual participants included in the study.

Availability of data and material The datasets analysed during the current study is available from the corresponding author on reasonable request.
Funding This study was supported by an unrestricted grant from Sanofi.

Open Access This article is distributed under the terms of the Creative Commons Attribution 4.0 International License (http://creativecommons.org/licenses/by/4.0/), which permits unrestricted use, distribution, and reproduction in any medium, provided you give appropriate credit to the original author(s) and the source, provide a link to the Creative Commons license, and indicate if changes were made.

\section{References}

1. UK Hypoglycaemia Study Group (2007) Risk of hypoglycaemia in types 1 and 2 diabetes: effects of treatment modalities and their duration. Diabetologia 50(6):1140-1147

2. Williams SA, Shi L, Brenneman SK, Johnson JC, Wegner JC, Fonseca V (2012) The burden of hypoglycemia on healthcare utilization, costs, and quality of life among type 2 diabetes mellitus patients. J Diabetes Complicat 26(5):399-406

3. Marrett E, Radican L, Davies MJ, Zhang Q (2011) Assessment of severity and frequency of self-reported hypoglycemia on quality of life in patients with type 2 diabetes treated with oral antihyperglycemic agents: a survey study. BMC Res Notes 4(1):251

4. Williams SA, Pollack MF, DiBonaventura M (2011) Effects of hypoglycemia on health-related quality of life, treatment satisfaction and healthcare resource utilization in patients with type 2 diabetes mellitus. Diabetes Res Clin Pract 91(3):363-370

5. Green AJ, Fox KM, Grandy S, Group SS (2012) Self-reported hypoglycemia and impact on quality of life and depression among adults with type 2 diabetes mellitus. Diabetes Res Clin Pract 96(3):313-318

6. Barendse S, Singh H, Frier B, Speight J (2012) The impact of hypoglycaemia on quality of life and related patient-reported outcomes in type 2 diabetes: a narrative review. Diabet Med 29(3):293-302

7. Cox DJ, Irvine A, Gonder-Frederick L, Nowacek G, Butterfield J (1987) Fear of hypoglycemia: quantification, validation, and utilization. Diabetes Care 10(5):617-621

8. Hajos T, Pouwer F, De Grooth R, Holleman F, Twisk J, Diamant $M$ et al (2011) Initiation of insulin glargine in patients with type 2 diabetes in suboptimal glycaemic control positively impacts health-related quality of life. A prospective cohort study in primary care. Diabet Med 28(9):1096-1102

9. Hartman I (2008) Insulin analogs: impact on treatment success, satisfaction, quality of life, and adherence. Clin Med Res 6(2):54-67

10. Heinemann L, Linkeschova R, Rave K, Hompesch B, Sedlak MART, Heise T (2000) Time-action profile of the long-acting insulin analog insulin glargine (HOE901) in comparison with those of NPH insulin and placebo. Diabetes Care 23(5):644-649

11. Horvath K, Jeitler K, Berghold A, Ebrahim SH, Gratzer TW, Plank J et al (2007) Long-acting insulin analogues versus NPH insulin (human isophane insulin) for type 2 diabetes mellitus. Cochrane Libr (2):CD005613

12. Inzucchi SE, Bergenstal RM, Buse JB, Diamant M, Ferrannini E, Nauck M et al (2015) Management of hyperglycemia in type 2 diabetes, 2015: a patient-centered approach: update to a position statement of the American Diabetes Association and the European Association for the Study of Diabetes. Diabetes Care 38(1):140-149

13. World Health Organization (1998) Wellbeing measures in primary health care: the DepCare project: report on a WHO meeting 
Stockholm, Sweden 12-13 February 1998. WHO Regional Office for Europe, Copenhagen

14. Arbuckle RA, Humphrey L, Vardeva K, Arondekar B, DantenViala M, Scott JA et al (2009) Psychometric evaluation of the diabetes symptom checklist-revised (DSC-R)-A measure of symptom distress. Value Health 12(8):1168-1175

15. Harris S, Mamdani M, Galbo-Jørgensen CB, Bøgelund M, Gundgaard J, Groleau D (2014) The effect of hypoglycemia on healthrelated quality of life: Canadian results from a multinational time trade-off survey. Can J Diabetes 38(1):45-52

16. Gilet H, Gruenberger J-B, Bader G, Viala-Danten M (2012) Demonstrating the burden of hypoglycemia on patients' quality of life in diabetes clinical trials: measurement considerations for hypoglycemia. Value Health 15(8):1036-1041

17. Zammitt NN, Frier BM (2005) Hypoglycemia in type 2 diabetes pathophysiology, frequency, and effects of different treatment modalities. Diabetes Care 28(12):2948-2961
18. Chelliah A, Burge MR (2004) Hypoglycaemia in elderly patients with diabetes mellitus. Drugs Aging 21(8):511-530

19. Boyle PJ, Zrebiec J (2007) Physiological and behavioral aspects of glycemic control and hypoglycemia in diabetes. South Med J 100(2):175-182

20. Murata GH, Duckworth WC, Shah JH, Wendel CS, Hoffman RM (2004) Factors affecting hypoglycemia awareness in insulintreated type 2 diabetes: the Diabetes Outcomes in Veterans study (DOVES). Diabetes Res Clin Pract 65(1):61-67

21. Donnelly LA, Morris AD, Frier BM, Ellis JD, Donnan PT, Durrant $R$ et al (2005) Frequency and predictors of hypoglycaemia in type 1 and insulin-treated type 2 diabetes: a population-based study. Diabet Med 22(6):749-755

22. Schafer JL, Graham JW (2002) Missing data: our view of the state of the art. Psychol Methods 7(2):147 